\title{
MICROBIAL LEAKAGE AND APICAL INFLAMMATORY RESPONSE IN DOG'S TEETH AFTER ROOT CANAL FILLING WITH DIFFERENT SEALERS, POST SPACE PREPARATION AND EXPOSURE TO THE ORAL ENVIRONMENT
}

\author{
Maximiliano Schünke GOMES ${ }^{1}$, Fernando Branco BARLETTA ${ }^{2}$, Alvaro DELLA BONA ${ }^{3}$, \\ José Roberto VANNI ${ }^{4}$, Charles da Cunha PEREIRA ${ }^{5}$, José Antônio Poli de FIGUEIREDO ${ }^{6}$
}

\author{
1- DDS, MSc in Endodontics, Captain of the Medical and Dental Center of the Health Department at Brigada Military, RS, Brazil. \\ 2- DDS, PhD, Associate Professor of the Department of Dentistry and Endodontics and Postgraduate Program,Lutheran University of Brazil, \\ Canoas, RS, Brazil and School of Dentistry, University of Santa Cruz do Sul, Santa Cruz do Sul, RS, Brazil. \\ 3- DDS, MMedSci, PhD, Senior Professor, Dental School, University of Passo Fundo (UPF), Passo Fundo, RS, Brazil. \\ 4- DDS, PhD, Chair Professor, Dental School, University of Passo Fundo (UPF), Passo Fundo, RS, Brazil. \\ 5- DDS, MSc, Associate Professor, Dental School, Lutheran University of Brazil (ULBRA), Cachoeira do Sul, RS, Brazil. \\ 6- DDS, MSc, PhD, Clinical Lecturer in Endodontology, Unit of Endodontology of Eastman Dental Institute, UCL.
}

Corresponding address: Maximiliano Schünke Gomes - Rua Ramiro Barcelos, 1172 / cj.212 - 90035-002 - Porto Alegre, RS, Brazil - Phone: +55 51 3311-8571 / 9956-9626. Fax: +55 51 3225-8796 - e-mail: endomax@terra.com.br

Received: January 23, 2007 - Modification: August 8, 2007 - Accepted: August 20, 2007

\begin{abstract}
C

oronal leakage is an important factor affecting the outcome of endodontic therapy. This study evaluated the microbial leakage (ML) and the apical inflammatory response (AIR) in dog's teeth after root canal filling with three endodontic sealers, post preparation and exposure to the oral environment, testing the hypothesis that there is a positive correlation between these two histological parameters (ML and AIR). Sixty-four root canals of 8 mongrel dogs were cleaned, shaped and randomly distributed into groups according to the sealer to be used: Sealer $26(\mathrm{n}=18)$; AH Plus $(\mathrm{n}=18)$; RoekoSeal $(\mathrm{n}=19)$; no sealer control group $(\mathrm{n}=9)$. Root canals were filled by the lateral condensation technique. Post space preparation left $4 \mathrm{~mm}$ of filling material in the apical root third, and specimens were exposed to the oral environment for 90 days. The dogs were killed and jaw blocks were histologically processed using Brown \& Brenn and HE staining techniques. ML and AIR were scored from 1 to 4 . Results were analyzed statistically using ANOVA, Duncan's post-hoc test and Spearman's correlation. ML and AIR score

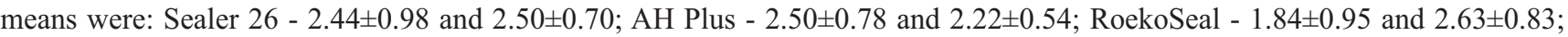
Control $-2.56 \pm 1.23$ and $3.11 \pm 0.60$. Statistically significant differences in AIR scores were found between the AH Plus and control groups $(\mathrm{p}<0.05)$. Although RoekoSeal had the lowest ML means, and AH Plus, the lowest AIR means after 90-day exposure to the oral environment, no statistically significant differences were found between the three sealers under study, and no correlation was found between ML and AIR.
\end{abstract}

Uniterms: Dental leakage; Periapical periodontitis; Root canal filling materials; Post and core technique.

\section{INTRODUCTION}

The main purpose of endodontic therapy is to eliminate infection from the root canal system and to prevent its reinfection in such a way that the health of periapical tissues is restored and maintained ${ }^{16}$.

Obturating methods and materials capable of tightly sealing the root canal system and blocking the communication of the periapical region with the oral environment and its potential irritants have been intensively pursued by clinicians and researchers. Several studies have been carried out to develop techniques and materials capable of preventing coronal leakage along endodontic fillings $s^{7,9,12,14,23}$. Such investigative effort is justified by the important role that leakage through obturation may play on the prognosis of endodontic therapy.

Studies have confirmed that endodontic failure is often associated with coronal sealing ${ }^{22}$, the consequent penetration of microorganisms $s^{5,26}$ and their toxins ${ }^{27}$ along throughout fillings, and their potentially deleterious effects on periapical tissues ${ }^{16,17,25}$.

Such situation is even more concerning when an 
intraradicular post space has to be prepared after completion of an endodontic treatment, further reducing the amount of filling material in the apical region of the canal and, therefore, reducing the physical barrier between the oral environment and periapical tissues. A large number of studies have investigated this problem ${ }^{1,3,15,21,29,30}$. However, there remains a disturbing distance between information provided by these investigations and its actual clinical implications because most of these studies follow an in vitro methodology.

Few in vivo studies have investigated the problem of

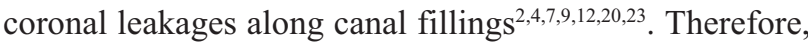
the purpose of this study was to histologically evaluate, using the Brown \& Brenn and hematoxylin-eosin staining methods, the degree of microbial leakage (ML) and the apical inflammatory response (AIR) of dog's teeth after filling of root canals with different endodontic sealers (Sealer 26, AH Plus and RoekoSeal), post space preparation, and exposure to the oral environment, testing the hypothesis that there is a positive correlation between these two histological parameters (ML and AIR).

\section{MATERIAL AND METHODS}

This study was approved by the Ethics in Research Committees of fhe Lutheran University of Brasil (ULBRA) and University of Passo Fundo (UPF).

The study sample was composed of 64 roots from lower second, third and fourth premolars and upper right second and third premolars from 8 small dogs of undefined breed from the UPF animal care facility.

The dogs received general anesthesia, as described by Kopper, et al. ${ }^{9}$ (2003), and the teeth to be treated were isolated with a rubber dam. Occlusal coronal access was performed with a \# 1012 high-speed round diamond bur (Fava Ind. e Com. Ltda., Francisco da Rocha, SP, Brazil) under water cooling. A dental tissue bridge was kept between the two canal orifices. Working length was established at $1 \mathrm{~mm}$ short of the radiographic apex.

The teeth were prepared with hand instrumentation technique using \#15-40 21-mm-long K-files (Dentsply, Maillefer S.A., Ballaigues, Switzerland). The last instrument was a \#40 file so that the enlargement of all canals was standardized. Canals were irrigated at each instrument change with $2.00 \mathrm{~mL} \mathrm{1 \%}$ sodium hypochlorite (Novaderme - Farmácia de Manipulação, Santa Maria, RS, Brazil) and $17 \%$ trisodium EDTA (Novaderme - Farmácia de Manipulação, Santa Maria, RS, Brazil); the last irrigation was performed with $1 \%$ sodium hypochlorite.

After root canal instrumentation, access to the radiographic apex was performed to $1 \mathrm{~mm}$ beyond the working length, with a \# 20 file to facilitate the anatomic communication between the inside of the root canal and periapical tissues. The canals were dried with absorbing paper points (EndoPoints, Paraíba do Sul, RJ, Brazil) and obturated with master and accessory gutta-percha cones (EndoPoints, Paraíba do Sul, RJ, Brazil) using the lateral condensation technique, and one of three endodontic sealers: Sealer 26 (Dentsply, Petrópolis, RJ, Brazil; batch: 55637) - $(\mathrm{n}=18)$; AH Plus (Dentsply, Konstanz, Germany; batch: 0210000896) - ( $\mathrm{n}=18)$; and RoekoSeal (Roeko, Langenau, Germany; batch: 002) - (n=19).

All sealers were handled according to manufacturer's recommendations. In the control group, nine root canals were obturated only with master and accessory gutta-percha cones, without any endodontic sealer. The selection of sealer to be used in each canal was made by stratified randomization to ensure a uniform distribution of sealers among the dental groups and among the different dogs.

Post space was prepared by partial removal of filling material from the canal with \# 2 Largo burs (Dentsply, Maillefer, Ballaigues, Switzerland) until $4 \mathrm{~mm}$ of sealing material remained in the apical third of all canals. The teeth were temporarily sealed with glass ionomer cement (Vidrion R - SS White, Rio de Janeiro, RJ, Brazil) until setting of endodontic sealers.

An experienced endodontist was the single operator in charge of all clinical procedures, including coronal access, canal preparation, obturation and post-space preparation.

Twenty-four hours after these procedures, the dogs were sedated with an IM injection of 0.3 to $1.5 \mathrm{mg} / \mathrm{kg}$ xylazine hydrochloride (Virbaxyl 2\%, Virbac Ltda., São Paulo, SP, Brazil) and 5 to $10 \mathrm{mg} / \mathrm{kg}$ ketamine hydrochloride (Vetanarcolâ 5\%, Laboratórios König S. A., Avellaneda, Argentina) applied in the same syringe. Coronal sealing was then removed with a \#1012 high-speed diamond bur under cooling so that experimental teeth were exposed to the oral environment for 90 days.

After 90 days, the dogs were anesthetized and sacrificed with an intravenous $25 \mathrm{mg} / \mathrm{kg}$ anesthetic dose of sodium thiopental (Thiopentaxâ, Cristália, SP, Brazil) and $10 \mathrm{~mL} \mathrm{10 \%}$ potassium chloride (Aster, Sorocaba, SP, Brazil) to cause cardiac arrest. To obtain adequate fixation of tissues, 10\% formaldehyde was administered intravenously. Maxillas and mandibles were removed, labeled and stored in $10 \%$ formaldehyde until histological processing.

Histologic examination followed the routine procedures of the Histology Laboratory of ULBRA. To initiate decalcification, maxillas and mandibles were immersed in $45 \%$ formic acid with $16.67 \%$ sodium citrate solution, renewed every week, and kept in a plaster vibrator-mixer for 120 days. After that, the mandibles and maxillas were sectioned to separate the bone blocks of each of the dental roots, which were identified individually.

The specimens underwent alcohol baths at increasing concentration (95 to 100\%), clarified with xylol, and embedded in paraffin. The specimens were cut with a microtome (Leica RM-2165, Nussloch, Germany) to obtain longitudinal sections that reached the periapical region along the long axis of the tooth. Semi-serial 6-mm thick sections were obtained from each specimen. The slides were then stained with hematoxylin-eosin (HE) or Brown \& Brenn (B\&B), alternately.

Slides were analyzed under an optical microscope (Olympus BX-41, Olympus Optical Company Ltda., Japan) at progressive magnifications of 40, 100, 200, 400 and 1000X. 
An experienced examiner blindly analyzed the HE-stained slides of all specimens. To investigate periapical tissue inflammation after the different treatments protocols, the specimens were evaluated and classified as follows: Score 1 - no inflammation; Score 2 - sparse mononuclear cells; Score 3 -infiltrate of mononuclear cells and/or sparse neutrophils or eosinophils; Score 4 - infiltrate of polymorphonuclear neutrophils or eosinophils, with abscess areas;

A second blinded trained examiner analyzed the B\&Bstained slides. For the analysis of microbial leakage in the post-space lumen, obturating mass, dentinal tubules, lateral and accessory canals, apical lacunae and ramifications, and apical cement, the following classification was established: Score 1-no microorganisms; Score 2-microorganisms in the post-space lumen or in dentinal tubules surrounding the post space; Score 3 -microorganisms in dentinal tubules and/or post space walls surrounding remaining filling material; Score 4-microorganisms in the most apically filled area, in dentinal tubules and/or within lacunae and ramifications of apical cementum.

The examiners were instructed to consider, in each specimen, the histologic section with the highest or most severe degree of microbial leakage or tissue inflammation.

Statistical analysis of results was performed with the calculation of means and standard deviations of scores of periapical inflammation and microbial leakage in all groups. Groups were compared using ANOVA followed by identification of differences with the Duncan's post-hoc test. Spearman's correlation was used for the analysis of correlations between scores of apical inflammatory response (AIR) and microbial leakage (ML) of all specimens. The level of significance was established at 0.05 .

\section{RESULTS}

Table 1 shows the score means and standard deviations of microbial leakage (ML) and apical inflammatory response (AIR) for the Sealer 26, AH Plus, RoekoSeal and control groups. There was statistically significant difference between the mean scores of inflammatory response in the
AH Plus and control groups. No statistically significant difference was found in the analysis of microbial leakage, although the RoekoSeal group had the lowest mean scores.

The results of Spearman correlation $(\mathrm{r}=0.039)$ did not show any statistically significant correlation between the microbial leakage (ML) scores and the apical inflammatory response (AIR). In the analysis of intraobserver agreement, Kappa was 0.781 for the specimens re-evaluated for apical inflammatory response and 0.605 for the re-evaluation of specimens analyzed for microbial leakage (B\&B).

Figures 1 to 6 show some examples of scores obtained in the evaluation of apical inflammatory response and microbial leakage in the different groups.

\section{DISCUSSION}

Of the several studies conducted to investigate coronal leakage, most followed an in vitro methodology using different leakage markers, such as radioisotopes ${ }^{14,15}$, dye penetration $^{21}$, fluid transport ${ }^{29,30}$, and others ${ }^{11}$. These laboratory studies cannot simulate the complex biological conditions of the oral environment and endodontic and periapical microenvironments, and, therefore, there remains a distance between information provided by these studies and their actual clinical meaning ${ }^{18}$.

Studies that use animal models are closer to clinical conditions because they take into consideration the effect of microbial associations and the variations of saliva flow and composition, and also because the control of important variables, such as observation time, operator, materials and techniques, is possible.

The chemical and mechanical preparation followed the step-back technique because all root canals were straight. Similarly to other studies ${ }^{2,5,7,12,23,26}$, the final instrument was \#40 to obtain a standardized enlargement of the canals of all specimens. Irrigation during preparation alternated $1 \%$ sodium hypochlorite and $17 \%$ EDTA to remove the smear layer at the same time of instrumentation, therefore reducing operation time.

An apical opening had to be performed because of the

TABLE 1- Means and standard deviation and statistical grouping of the microbial leakage (ML) and apical inflammatory response (AIR) between Sealer 26, AH Plus, RoekoSeal and Control groups

\begin{tabular}{llll} 
Experimental Group & N & \multicolumn{2}{c}{$\begin{array}{c}\text { Mean } \pm \text { Standard deviation } \\
\text { AL }\end{array}$} \\
\hline Sealer 26 & 18 & $2.44 \pm 0.98$ & $2.50^{\mathrm{ab} \pm 0.70}$ \\
AH Plus & 18 & $2.50 \pm 0.78$ & $2.22^{\mathrm{a}} \pm 0.54$ \\
RoekoSeal & 19 & $1.84 \pm 0.95$ & $2.63^{\mathrm{ab}} \pm 0.83$ \\
Control & 9 & $2.56 \pm 1.23$ & $3.11^{\mathrm{b}} \pm 0.60$ \\
& 64 & & 0.023 \\
\hline
\end{tabular}

$\left(^{*}\right)$ ANOVA, $\alpha=0.05$. Different letters indicate statistically significant differences (Duncan's post-hoc test). 

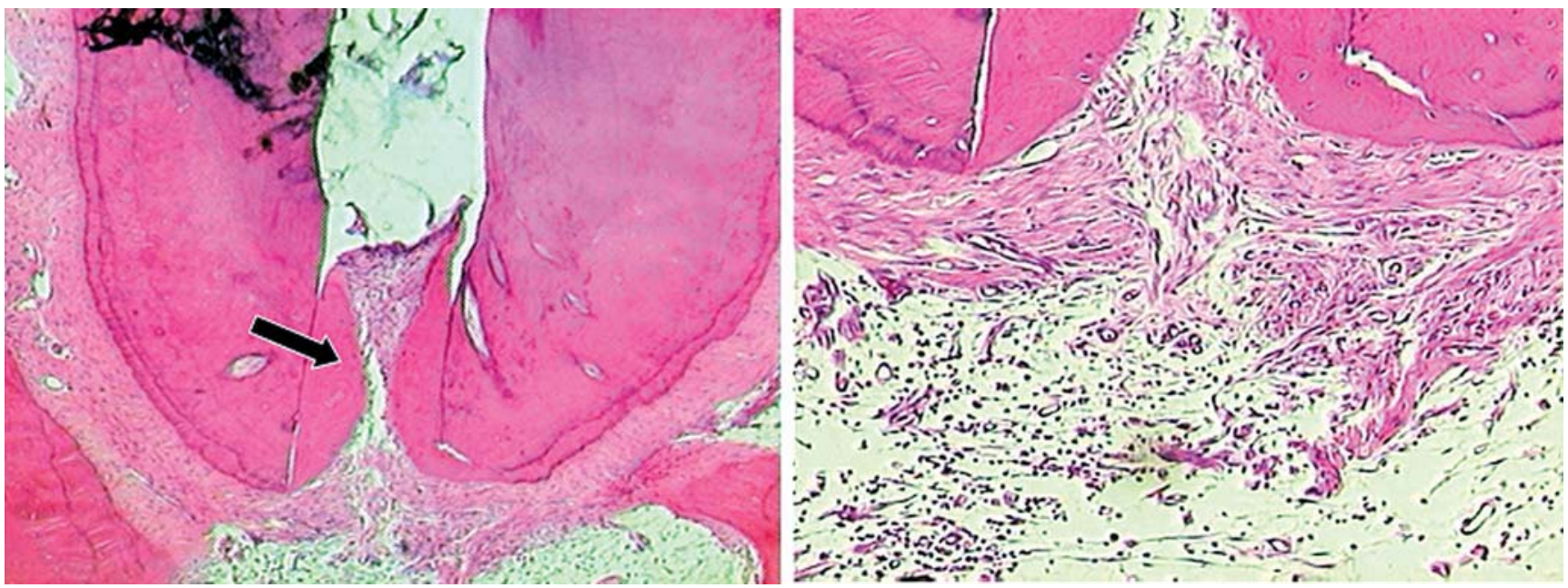

FIGURE 1- A - SEM micrograph of apical and periapical regions of a specimen in the AH Plus group. Observe newly formed hard tissue deposition (arrow), followed by an organized periodontal ligament. HE. 40X. B - Close-up view. Observe sparse mononuclear cells, compatible with score 2 of AIR. HE. 100X
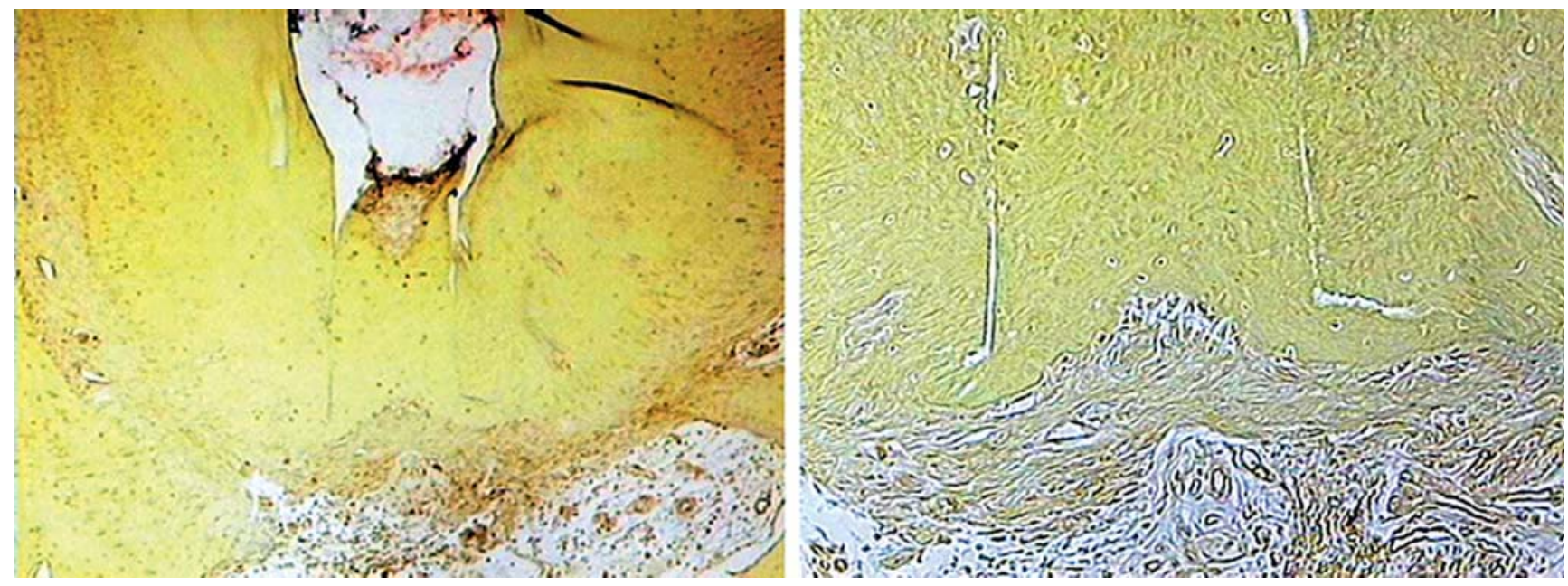

FIGURE 2- A - SEM micrograph of apical and periapical regions of the same specimen showed in Fig.1, belonging to the $\mathrm{AH}$ Plus group. No microorganisms can be seen (score 1 of ML). B\&B. 40X. B - Close-up view. B\&B. 100X
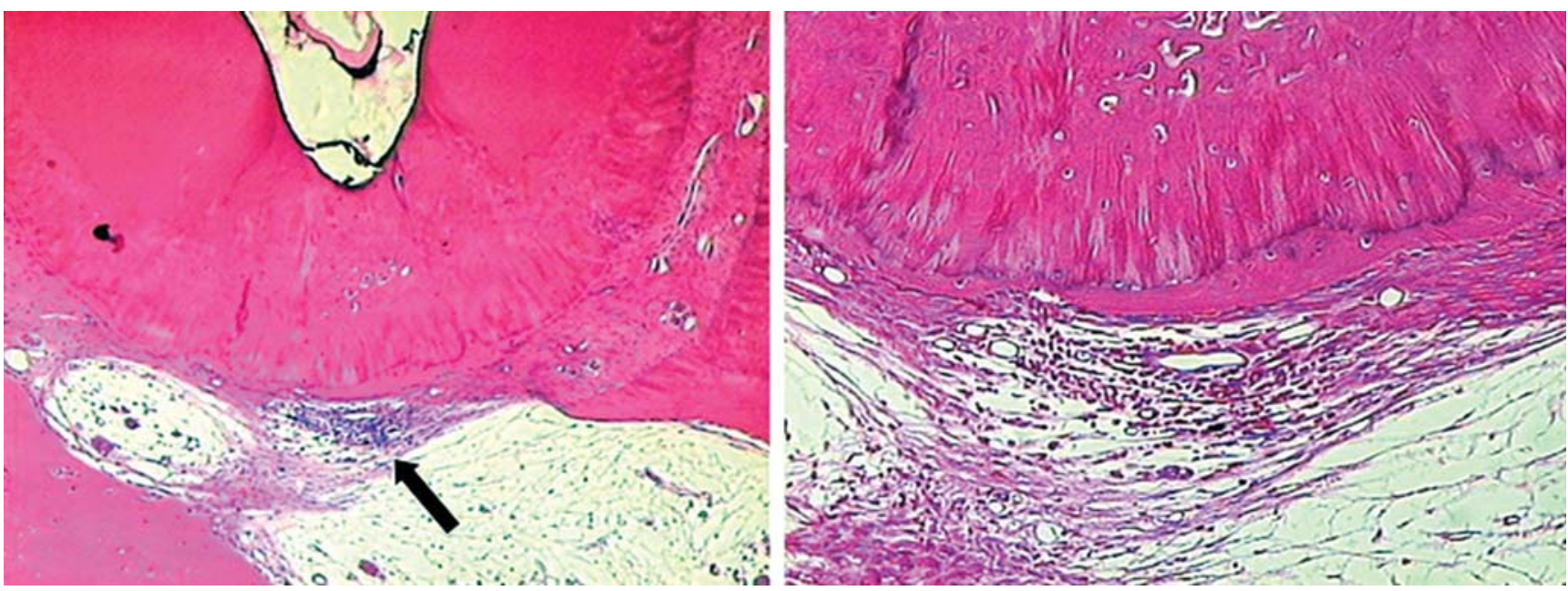

FIGURE 3- A - SEM micrograph of apical and periapical regions of a specimen belonging to the RoekoSeal group. Note inflammatory infiltrate along apical periodontal ligament (arrow). HE. 40X. B - Close-up view. Mononuclear inflammatory reaction can be observed, compatible with score 3 of AIR. H\&E. 100X 


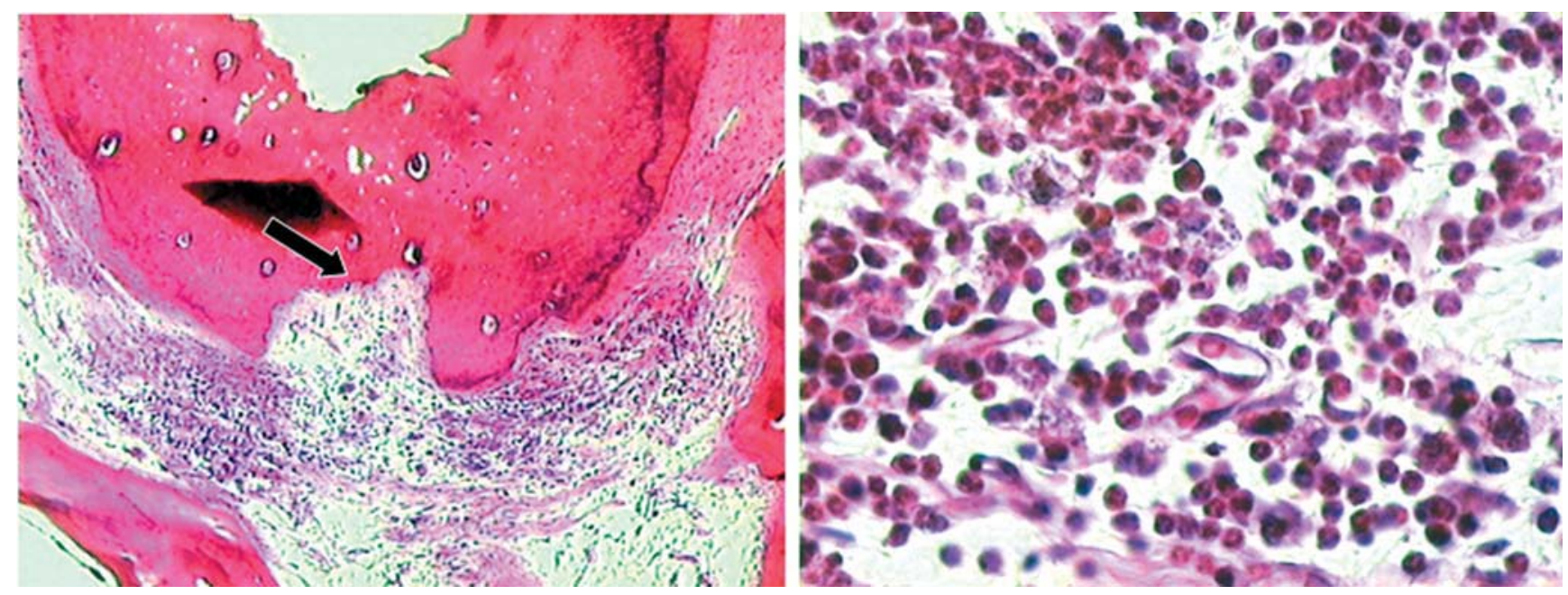

FIGURE 4- A - SEM micrograph of apical and periapical regions of a specimen in the control group. Note inflammatory infiltrate along apical periodontal ligament. Areas of apical cementum and dentin resorption can be seen (arrow), as well as resorption of adjacent bone. HE. 40X. B - Close-up view. Intense neutrophil infiltrate with some dead cells and resulting in pus formation, compatible with score 4 of AIR. HE. 400X
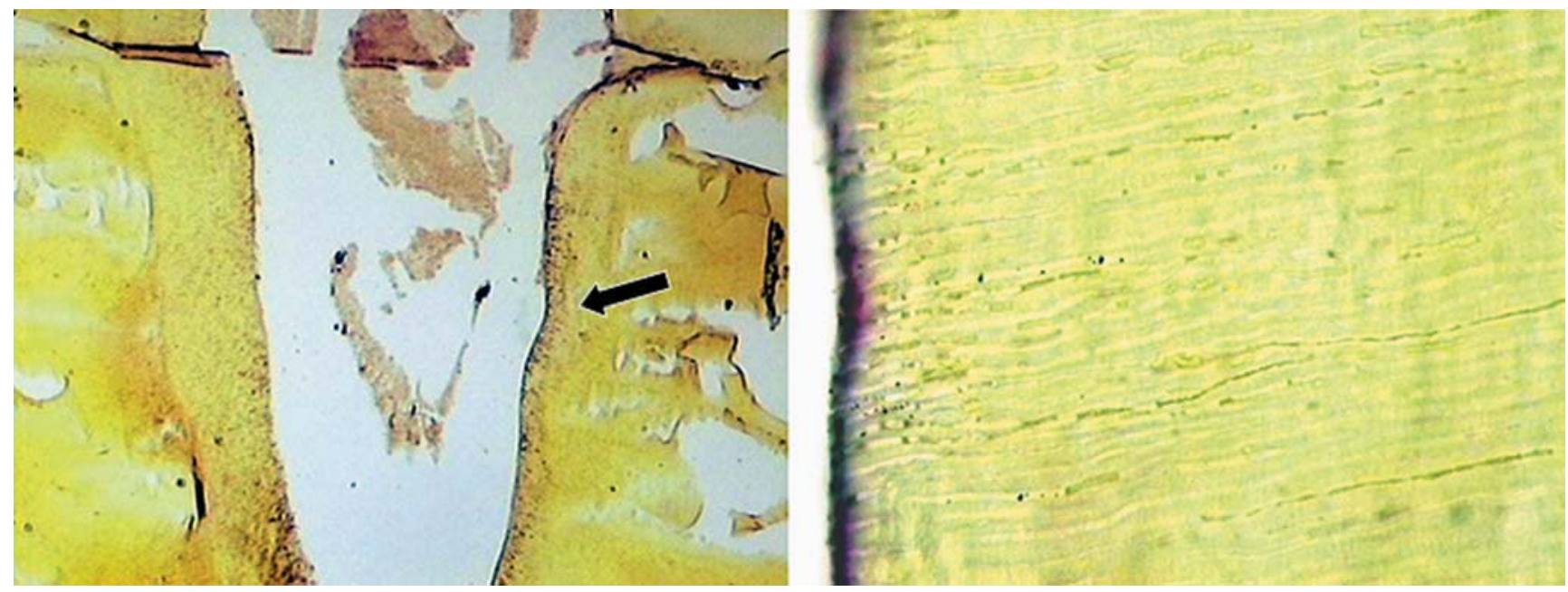

FIGURE 5- A - SEM micrograph of cervical region of the same specimen shown in Fig. 4, belonging to the control group. Observe the presence of microorganisms within lumen, canal walls and dentinal tubules (arrow). B\&B. 40X. B - Close-up view. B\&B. 400X
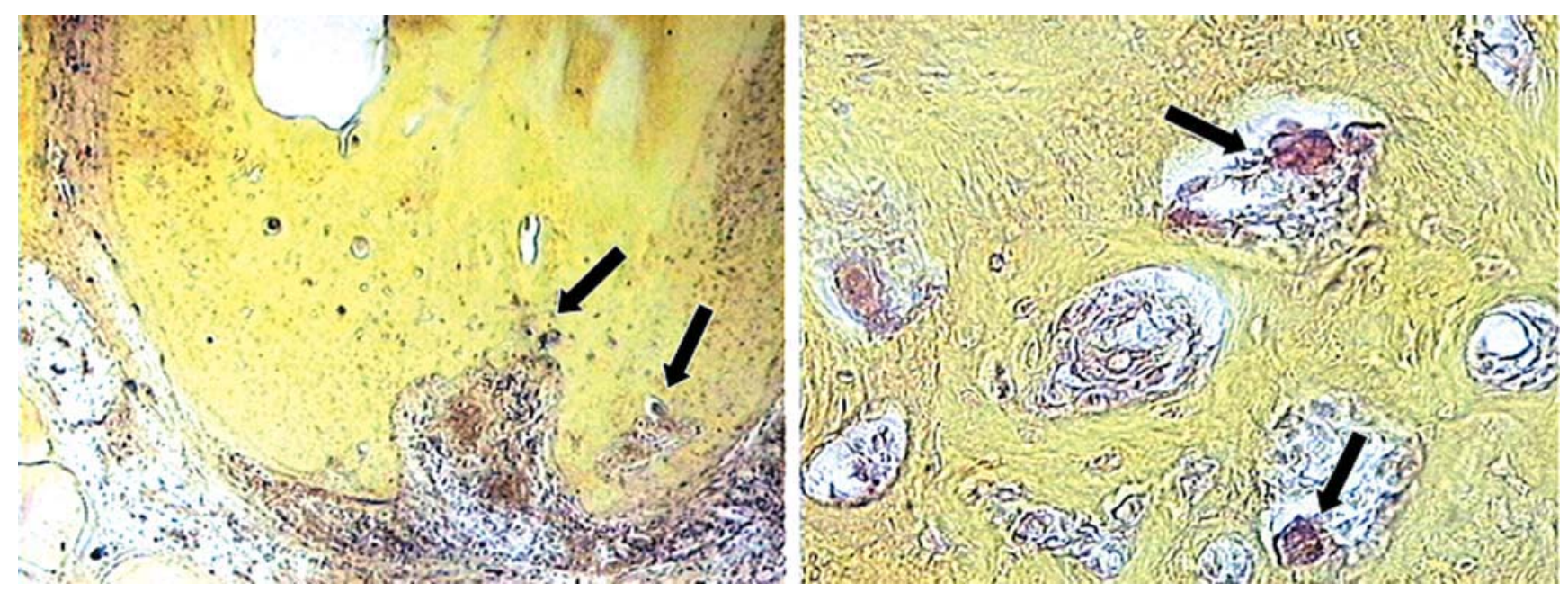

FIGURE 6- A - SEM micrograph of apical and periapical regions of the same specimen of Figs. 4 and 5 . This specimen scored 4 in ML. Note presence of microorganisms within apical lacunae (arrows) near root resorption area. B\&B. 40X. B Close-up view. Microorganisms (arrows) within lacunae and ramifications of apical cementum. B\&B. 400X 
prevalence of apical ramifications and the absence of a main foramen in the dogs' teeth. The diameter of the apical opening was standardized using a \# 20 file, and the communication between the canal space and the periapical tissues was established in an attempt to reproduce as close as possible the actual conditions of human teeth. This surgical step was not adopted in other studies ${ }^{2,23}$.

Root canals were obturated with the lateral condensation technique, in agreement with a large number of studies $^{2,5,7,9,12,21,26,30}$. The length of obturation remaining after post space preparation is a factor of great importance. It is clear in the literature ${ }^{15}$ that the length of the obturation is positively correlated with its sealing ability. Therefore, we decided to standardize the length of remaining obturation in all specimens although the working length was not the same in all roots. This was undertaken according to other studies ${ }^{1,2,3}$, which used a mean 4-mm length of remaining obturating material, and adopted the same length as that used in this study. Moreover, this length is very close to the clinically adopted lower threshold for apical obturation when an intraradicular post is used.

In this study, root fillings were exposed to the oral environment for 90 days, a period of time also used in other studies $2,3,5,13,23$. It was not the purpose of this study to analyze inflammatory response or microbial leakage at different exposure times. The inclusion of study groups with different exposure times would require a substantially greater number of dogs, an ethical barrier in the current institutional reality. Other studies, however, should be conducted to investigate microbial leakage and tissue response at different time points without including type of endodontic cement as a variable.

The analysis of microbial leakage was conducted by means of analysis of B\&B-stained slides. However, this method is greatly limited for the detection of microorganisms. According to Nair, et al. ${ }^{17}$ (1990), the absence of fungi or bacteria in an optical microscopic examination does not necessarily mean their inexistence. Also, Magura, et al. ${ }^{13}$ (1991) did not find microorganisms in the histologic examination of their specimens although the microbiologic test was positive. Moreover, it is known that specimen decalcification destroys microorganisms or changes their staining properties ${ }^{28}$. Despite these difficulties, we believe that B\&B staining should be used to evaluate the presence of bacteria in experimental studies. Lanza $^{10}$ (2006), however, suggested that B\&B staining is not an accurate method and is not the most adequate to detect microorganisms in demineralized tissues, although it is the most often indicated and easily available staining method for the detection of microorganisms under optical microscopy. Therefore, the results obtained from the analysis of HE-stained slides, representative of the inflammatory response in the periapical region, provide methodologically sounder and more reliable results than the information obtained with B\&B staining. This, however, does not invalidate the results of microbial leakage obtained in this study.

RoekoSeal sealer had the best performance regarding microbial leakage (mean score $=1.81$ ), although this group also had the highest standard deviation (51\% of the mean) and statistical analysis did not show any significant difference. This finding is in agreement with those reported by $\mathrm{Wu}$, et al. ${ }^{30}$ (2002), who conducted an in vitro study and showed that RoekoSeal has a consistent sealing ability that was effective for 18 months. The study by Pereira ${ }^{20}$ (2007) found a lower sealing ability for RoekoSeal compared to EndoRes, but differences were not significant in the comparison to AH Plus. The direct comparison of our results to those reported by Pereira ${ }^{20}$ (2007) has limited value because the methodologies were substantially different, even though both studies were conducted with dogs. Likewise, the comparison to the results reported by Çobankara, et al. ${ }^{6}$ (2004) is limited because their in vitro study included AH 26 sealer, which was not used in the present study.

Some explanations may be suggested for the lower mean scores of microbial leakage in the RoekoSeal group. Firstly, the main content of this material is polydimethylsiloxane, that is, silicone, which gives the sealer good flowability and high penetrability even in dentinal tubules, as observed in the histologic examination. Moreover, RoekoSeal has excellent dimensional stability ${ }^{19}$ and is, therefore, superior to materials that contain resin in their composition - like Sealer 26 and AH Plus - and that may undergo certain polymerization shrinkage, which results in empty spaces in the obturating mass. Finally, the low solubility of RoekoSeal may also have contributed to the microbial leakage scores found in the present study.

There was a significant difference between mean inflammatory scores in the AH Plus group (2.22) and in the control group (3.11). This finding suggests that AH Plus is the sealer with the most favorable result of apical tissue response to exposure to oral environment. This result may be explained by the excellent biocompatibility of this material $^{8}$, as well as by a possible antimicrobial action ${ }^{24}$ against the microorganisms that leak into the obturating mass.

Studies by Kopper, et al. ${ }^{9}$ (2003), among others, found lower mean coronal leakage values for AH Plus. In our study, AH Plus mean microbial leakage scores were greater than those recorded for RoekoSeal. However, the effect of this possible leakage on periapical tissues was less aggressive in the AH Plus specimens. These data indicate that it was not possible to establish a correlation between the microbial leakage and apical inflammatory response scores, rejecting the study hypothesis of a positive correlation between the degree of leakage and the apical inflammatory response. However, in in vivo studies, the associations between aggression (organization, amount and virulence of microorganisms) and defense (host immune competence) are extremely rich and complex, and may lead to results incompatible with a Cartesian, simplistic logic, but understandable, nevertheless, in the light of biology.

The control group had the highest score means for both microbial leakage and apical inflammatory response. This result is in agreement with those of several other studies $^{12,14,27,30}$ that used control groups of teeth obturated 
with gutta-percha cones alone without endodontic sealer. These findings confirm the idea that endodontic sealers have an important role in the final sealing of root canals. It is important to notice that none of the sealers used in this study was capable of completely blocking leakage. Endodontic sealers can, at most, delay cervical leakage but not block it entirely. This finding is supported by the results of several studies ${ }^{2,9,20}$.

Finally, considering the materials examined in this work, it seems relevant to mention that the more favorable results of AH Plus for apical inflammatory response may be an important clinical indication that this material provides better chances of preserving periapical health in a situation of exposure to fluids and oral microbiota.

\section{CONCLUSIONS}

None of the endodontic sealers tested in this study blocked microbial leakage after 90 days of exposure to the oral environment. Although RoekoSeal showed the lowest microbial leakage means and AH Plus the lowest apical inflammatory response means, no statistically significant differences were found between the three sealers and no correlation was found between microbial leakage and apical inflammatory response, thus rejecting the testing hypothesis of this study.

\section{REFERENCES}

1- Alves J, Walton R, Drake D. Coronal leakage: endotoxin penetration from mixed bacterial communities through obturated, post-prepared root canals. J Endod. 1998; 24(9):587-91.

2- Barbosa HG, Holland R, de Souza V, Dezan Júnior E, Bernabé PFE, Otoboni Filho JA, et al. Healing process of dog teeth after post space preparation and exposition of the filling material to the oral environment. Braz Dent J. 2003;14(2):103-8.

3- Barrieshi KM, Walton RE, Johnson WT, Drake DR. Coronal leakage of mixed anaerobic bacteria after obturation and post space preparation. Oral Surg Oral Med Oral Pathol Oral Radiol Endod. 1997;84(3):310-4.

4- Bier CA, Figueiredo JA, Della Bona A, Kopper PMP, Vanni JR, Bopp S. In vivo analysis of post space sealing with different adhesive materials. J Appl Oral Sci. 2003;11(3):168-74.

5- Chailertvanitkul P, Saunders WP, MacKenzie D, Weetman DA. An in vitro study of the coronal leakage of two root canal sealers using an obligate anaerobe microbial marker. Int Endod J. 1996;29:249- 55

6- Çobankara FK, Adanir N, Belli S. Evaluation of the influence of smear layer on the apical and coronal sealing ability of two sealers. J Endod. 2004;30(6):406-9.

7- Friedman S, Komorowski R, Maillet W, Klimaite R, Nguyen HQ, Torneck CD. In vivo resistance of coronally induced bacterial ingress by an experimental glass ionomer cement root canal sealer. J Endod. $2000 ; 26(1): 1-5$
8- Hauman CH, Love RM. Biocompatibility of dental materials used in contemporary endodontic therapy: a review. Part 2. Root-canalfilling materials. Int Endod J. 2003;36(3):147-60.

9- Kopper PM, Figueiredo JA, Della Bona A, Vanni JR, Bier CA, Bopp S. Comparative in vivo analysis of the sealing ability of three endodontic sealers in post-prepared root canals. Int Endod J. 2003;36(12):857-63.

10 - Lanza LD, Silva GA, Lopes-Júnior N, Moreira A, Alves JB. Direct pulp capping with a dentin bonding system in human teeth: a clinical and histological evaluation. Oper Dent. 2006; 31(3):297-307.

11 - Lyroudia K, Pantelidou O, Mikrogeorgis G, Chatzikallinikidis C, Nikopoulos N, Pitas I. The use of 3D computerized reconstruction for the study of coronal microleakage. Int Endod J. 2000;33(3):2437

12- Madison S, Wilcox LR. An evaluation of coronal microleakage in endodontically treated teeth: Part III: In vivo study. J Endod. $1988 ; 14(9): 455-8$

13- Magura ME, Kafrawy AH, Brown CE Jr, Newton CW. Human saliva coronal microleakage in obturated root canals: an in vitro study. J Endod. 1991;17(7):324-31.

14- Marshall FJ, Massler M. The sealing of pulpless teeth evaluated with radioisotopes. J Dent Med. 1961;6(4):172-84.

15- Metzger Z, Abramovitz R, Abramovitz L, Tagger M. Correlation between remaining length of root canal fillings after immediate post space preparation and coronal leakage. J Endod. 2000;26(12):724-

16- Möller AJ, Fabricius L, Dahlen G, Ohman AE, Heyden G. Influence on periapical tissues of indigenous oral bacteria and necrotic pulp tissue in monkeys. Scand J Dent Res. 1981;89(6):475-84.

17- Nair PN, Sjogren U, Krey G, Kahnberg KE, Sundqvist G. Intraradicular bacteria and fungi in root-filled, asymptomatic human teeth with therapy-resistant periapical lesions: a long-term light and electron microscopic follow-up study. J Endod. 1990;16(12):580-8.

18- Oliver CM, Abbott PV. Correlation between clinical success and apical dye penetration. Int Endod J. 2001;34(8):637-44.

19- Orstavik D, Nordahl I, Tibballs JE. Dimensional change following setting of root canal sealer materials. Dent Mater. 2001;17:512-9.

20- Pereira CC, Oliveira EPM, Gomes MS, Della-Bona A, Vanni JR, Kopper PMP, Figueiredo JAP. Comparative in vivo analysis of the sealing ability of three endodontic sealers in dog teeth after postspace preparation. Aust Endod J. 2007 (in press) doi: 10.1111/ j.1747-4477.2007.00069.x

21 - Ravanshad S, Torabinejad M. Coronal dye penetration of the apical filling materials after post space preparation. Oral Surg Oral Med Oral Pathol. 1992;74(5):644-7.

22- Ray HA, Trope M. Periapical status of endodontically treated teeth in relation to the technical quality of the root filling and the coronal restoration. Int Endod J. 1995;28(1):12-8.

23-Shipper G, Teixeira FB, Arnold RR, Trope M. Periapical inflammation after coronal microbial inoculation of dog roots filled with gutta-percha or resilon. J Endod. 2005 Feb;31(2):91-6.

24- Siqueira JF Jr, Favieri A, Gahyva SM, Moraes SR, Lima KC, Lopes HP. Antimicrobial activity and flow rate of newer and established root canal sealers. J Endod. 2000;26(5):274-7. 
25 - Sundqvist G, Figdor D, Persson S, Sjogren U. Microbiologic analysis of teeth with failed endodontic treatment and the outcome of conservative re-treatment. Oral Surg Oral Med Oral Pathol. 1998;85(1):86-93.

26- Torabinejad M, Ung B, Kettering JD. In vitro bacterial penetration of coronally unsealed endodontically treated teeth. J Endod. 1990;16(12):566-9.

27- Trope M, Chow E, Nissan R. In vitro endotoxin penetration of coronally unsealed endodontically treated teeth. Endod Dent Traumatol. 1995;11(2):90-4.

28- Wijnbergen M, Van Mullem PJ. Effect of histological decalcifying agents on number and stainability of gram-positive bacteria. J Dent Res. 1987;66(5):1029-31.

29- Wu MK, De Gee AJ, Wesselink PR, Moorer WR. Fluid transport and bacterial penetration along root canal fillings. Int Endod J. $1993 ; 26(4): 203-8$.

30- Wu MK, Tigos E, Wesselink PR. An 18-month longitudinal study on a new silicon based sealer, RSA RoekoSeal: a leakage study in vitro. Oral Surg Oral Med Oral Pathol. 2002;94(4):499-502. 